\title{
BMJ Open Initial use of supplementary oxygen for trauma patients: a systematic review
}

To cite: Eskesen TG, Baekgaard JS, Steinmetz J, et al. Initial use of supplementary oxygen for trauma patients: a systematic review. BMJ Open 2018;8:e020880. doi:10.1136/ bmjopen-2017-020880

- Prepublication history for this paper is available online. To view these files, please visit the journal online ( $h$ ttp://dx.doi. org/10.1136/bmjopen-2017020880).

Received 1 December 2017 Revised 20 April 2018 Accepted 5 June 2018
Check for updates

Department of Anesthesia, University of Copenhagen, Copenhagen, Denmark

Correspondence to Trine Grodum Eskesen; trinegeskesen@live.dk

Trine Grodum Eskesen, Josefine Stokholm Baekgaard, Jacob Steinmetz, Lars S Rasmussen

\section{ABSTRACT}

Objective This systematic review aimed to identify and describe the evidence for supplementary oxygen for spontaneously breathing trauma patients, and for high $(0.60-0.90)$ versus low $(0.30-0.50)$ inspiratory oxygen fraction $\left(\mathrm{FiO}_{2}\right)$ for intubated trauma patients in the initial phase of treatment.

Methods Several databases were systematically searched in September 2017 for studies fulfilling the following criteria: trauma patients (Population); supplementary oxygen/high $\mathrm{FiO}_{2}$ (Intervention) versus no supplementary oxygen/low FiO ${ }_{2}$ (Control) for spontaneously breathing or intubated trauma patients, respectively, in the initial phase of treatment; mortality, complications, days on mechanical ventilation and/or length of stay (LOS) in hospital/intensive care unit (ICU) (utcomes); prospective interventional trials (Study design). Two independent reviewers screened and identified studies and extracted data from included studies. Results 6142 citations were screened with an inter-rater reliability (Cohen's kappa) of 0.88 . One interventional trial of intubated trauma patients was included. 68 trauma patients were randomised to receive an $\mathrm{FiO}_{2}$ of 0.80 (intervention group) or 0.50 (control group) during mechanical ventilation (first 6 hours). There was no significant difference in hospital or ICU LOS between the groups. No patient died in either group. Another interventional trial, not strictly fulfilling the inclusion criteria, was presented for descriptive purposes. 21 trauma patients were alternately assigned to two types of mechanical ventilation (first 48 hours), both aiming at an $\mathrm{FiO}_{2}$ of 0.40 , but resulted in estimated mean $\mathrm{FiO}_{2} \mathrm{~S}$ of 0.45 (intervention group) and 0.60 (control group). No difference in days on mechanical ventilation was found. Two patients in the control group died, none in the intervention group. No prospective, interventional trials on spontaneously breathing trauma patients were identified.

Conclusions Evidence for the use of supplementary oxygen for spontaneously breathing trauma patients is lacking, and the evidence for low versus high $\mathrm{FiO}_{2}$ for intubated trauma patients is limited.

PROSPERO registration number 42016050552

\section{BACKGROUND}

Trauma is estimated to be the number one cause of death for persons between 1 and 44 years of age, ${ }^{1}$ and costs related to trauma are a significant economic burden to society. ${ }^{2}$ The initial (prehospital and early in-hospital) treatment of trauma patients can be
Strengths and limitations of this study

- The use of predefined Population, Intervention, Control, Outcomes, Study design criteria to assess for study eligibility.

- The use of a wide search string in multiple databases.

- The use of a structured screening and inclusion process, as well as data collection and risk of bias assessment by two independent authors.

- There is a possibility of missing unpublished studies which creates a potential publication bias.

- It is possible that we did not identify all relevant studies despite our systematic methodology.

crucial for the subsequent injury outcome, but current management is based on guidelines that are not generally well supported by evidence, ${ }^{13}$ as research in this setting is difficult to conduct for numerous reasons.

Oxygen is probably the most commonly administered drug both in the prehospital and emergency department setting, and several studies have found supplementary oxygen to be widely used in the prehospital treatment of trauma patients. ${ }^{4-6}$ Oxygen is cheap, easily administered and, at least for shorter time frames, widely believed to be without any risk of harm. Supplementary oxygen treatment is recommended internationally in both the Advanced Trauma Life Support (ATLS) manual and the PreHospital Trauma Life Support manual. ${ }^{3}$ This often leads to a 'default' administration of oxygen even without an indication. ${ }^{5}$ Supplementary oxygen treatment is provided to prevent or correct hypoxaemia, as this may cause tissue hypoxia with organ injury. However, supplementary oxygen introduces a risk of hyperoxaemia which is associated with a risk of complications, especially lung damage, and liberal use of oxygen is associated with greater morbidity and mortality in surgical patients and in patients with acute conditions like stroke, myocardial infarction and cardiac arrest (CA)..$^{7-10}$ 
In intubated patients, an inspiratory oxygen fraction $\left(\mathrm{FiO}_{2}\right)$ of $0.30-0.50$ is often used during mechanical ventilation. A high $\mathrm{FiO}_{2}(0.60-0.90)$ intraoperatively has been suggested to reduce the incidence of surgical site infection; however, a recent systematic review did not detect a beneficial effect. ${ }^{10-12}$

As the evidence behind the current trauma guidelines with regard to oxygen therapy is not clear, and excessive oxygen administration has been found to be harmful in other patient populations, we sought to perform a systematic review to identify and summarise the evidence for the use of supplementary oxygen for spontaneously breathing trauma patients, and the use of high $(0.60-0.90)$ versus low (0.30-0.50) $\mathrm{FiO}_{2}$ for intubated trauma patients.

\section{METHODS}

\section{Protocol and registration}

We conducted a systematic review following the recommendations by the Cochrane Collaboration ${ }^{13}$ and the Preferred Reporting Items for Systematic Reviews and Meta-Analyses (PRISMA) Statement. ${ }^{14}$ The protocol was completed following the PRISMA Protocols ${ }^{15}$ and was registered in the International Prospective Register of Systematic Reviews. ${ }^{16}$

\section{Eligibility criteria}

Inclusion of studies was based on the following predefined Population, Intervention, Control, Outcomes, Study design (PICOS) criteria: trauma patients $>17$ years of age (Population); supplementary oxygen (Intervention) versus no supplementary oxygen ( neously breathing trauma patients and/or high (0.600.90) (Intervention) versus low $(0.30-0.50)$ (Control) $\mathrm{FiO}_{2}$ for intubated trauma patients in the initial phase of treatment $(<24$ hours after the traumatic incident including both prehospital and in-hospital phases); all-cause mortality, in-hospital mortality, in-hospital complications, days on mechanical ventilation and/ or length of stay (LOS) in hospital/intensive care unit (ICU) (Outcomes); prospective interventional trials (randomised and non-randomised) (Study design). Observational studies, reviews, expert opinions, case reports, letters, abstracts and editorials were excluded. There was no restriction to language or year of publication. Potential eligible studies where the full text could not be found were excluded.

\section{Information sources and search methods}

We searched MEDLINE, EMBASE and the Cochrane Library from inception to 22 September 2016 using the following predefined search string (presented search strategy is from MEDLINE):

1. ((trauma) OR traumat*) OR traumatic injury

2. (((( (oxygen*) OR oxygen) OR oxygenation) OR supplemental oxygen) OR fio2) OR hyperox*

3. $(((((((30$ day mortality) OR mortal*) $)$ OR all cause mortality) OR complicat*) OR in-hospital mortality)
OR length of stay) OR LOS) OR hospital mortality[MeSH Terms]) OR mortality[MeSH Terms]

4. \#1 AND \#2 AND \#3

5. Filter: Humans

Modification of the search string was made to fit EMBASE and the Cochrane Library format, respectively. The search was updated on 3 September 2017, and no new studies were found.

\section{Study selection}

Two independent authors (TGE and JSB) screened titles and abstracts from the primary search in all three databases. Screening was performed using Covidence (an online program facilitating the production of systematic reviews developed by the Cochrane Group). ${ }^{17}$ Inter-rater reliability was calculated using Cohen's kappa statistics. Both authors evaluated relevant studies in full text independently. Disagreement was resolved by discussion. If agreement could not be reached, a senior author (JS or LSR) was involved. Bibliographies of included studies were reviewed for further potentially relevant studies (so-called 'snowballing').

\section{Data collection and data items}

Data extraction was performed by two authors (TGE, JSB) independently using predetermined forms and facilitated by the data-extraction tool in Covidence. Collected study characteristics included study setting and country, study period and publication year. Data on methods, population, interventions and outcomes included study design, blinding, aim of the study, inclusion and exclusion criteria, number of included patients, baseline characteristics (ie, age, gender, mechanism of injury), fraction of inspired oxygen and oxygenation assessment of the intervention and control group, respectively, as well as any of the predefined outcome measures (primary outcome measure: all-cause mortality at 30 days; secondary outcome measures: in-hospital mortality, in-hospital complications, days on mechanical ventilation and/or LOS in hospital/ ICU).

\section{Risk of bias assessment}

The quality of the included studies was assessed by two independent authors (TGE, JSB) using the Cochrane risk of bias assessment tool in Covidence ${ }^{18}$ which consists of seven specific domains (random sequence generation, allocation concealment, blinding of participants and personnel, blinding of outcome assessment, incomplete outcome data, selective reporting and other bias). In each domain, the study is judged to have a low, high or unclear risk of bias.

\section{Summary measures and synthesis of results}

This systematic review was expected to be a descriptive summary of the current evidence.

Patient and public involvement

There was no patient involvement in this study. 


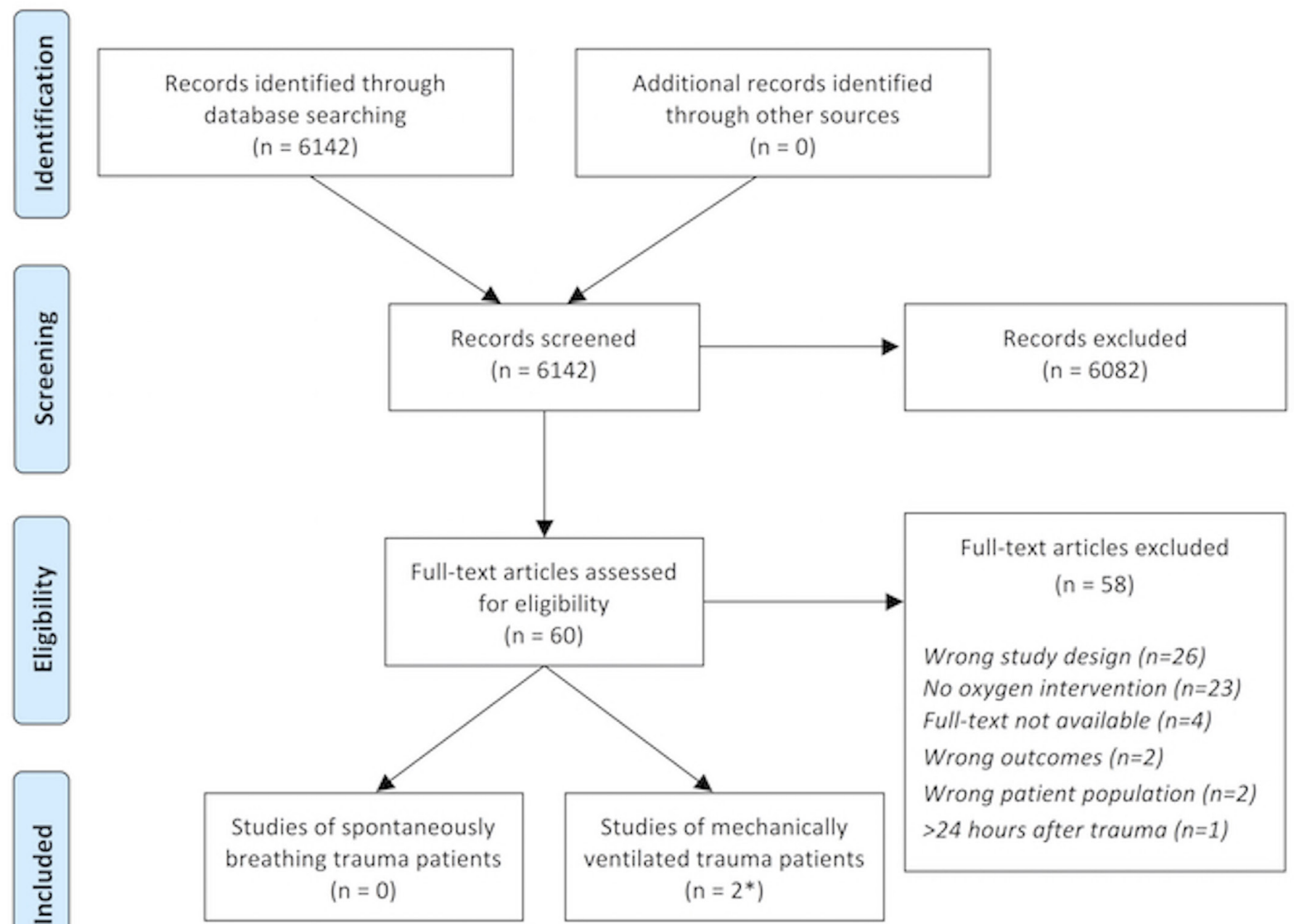

Figure 1 Preferred Reporting Items for Systematic Reviews and Meta-Analyses flow diagram of the identification, screening, eligibility and inclusion process. ${ }^{14}{ }^{*}$ One of the included studies ${ }^{20}$ did not strictly meet the inclusion criteria; however, it is included for descriptive purposes.

\section{RESULTS}

Our combined search strategy identified 6142 records to be considered for inclusion. After screening titles and abstracts, 60 articles were evaluated in full text for eligibility. An inter-rater reliability (Cohen's kappa) of 0.88 (CI: 0.82 to 0.94 ) for screening and selecting studies was obtained. After full-text review, only one study fulfilled the inclusion criteria and was included in the systematic review $^{19}$ (figure 1). Another study, which did not strictly fulfil the inclusion criteria, was also included for descriptive purposes. Both studies were prospective, interventional trials and included intubated trauma patients, and thus no prospective, interventional trials of spontaneously breathing trauma patients were identified. Characteristics, methods and results for the two included studies are summarised in table 1 .

Taher $e t a l^{19}$ performed a randomised study of 68 mechanically ventilated adult patients sustaining severe traumatic brain injury (TBI). The patients were randomised to receive an $\mathrm{FiO}_{2}$ of either 0.80 (intervention group) or 0.50 (control group) during the first 6 hours of treatment. A total of 34 patients in each group completed the study. The two groups were similar in terms of age, gender distribution and GCS on admission. Relevant outcomes for this systematic review were LOS in hospital and LOS in ICU. The study found no statistically significant difference between the intervention and control groups in either of these outcome measures (hospital LOS: 11.4 days (SD: 5.4) vs 13.9 days (SD: 8.1), respectively, $\mathrm{p}=0.14$; ICU LOS: 9.4 days (SD: 6.6 ) vs 11.4 days (SD: 8.4 ), respectively, $p=0.28$ ). No patients in either group died.

The study by Barzilay et $a l^{20}$ included 21 adult patients with chest trauma and severe respiratory insufficiency due to flail chest or pulmonary contusion requiring mechanical ventilation. Patients were alternately assigned to two different mechanical ventilation strategies: conventional mechanical ventilation or high-frequency positive pressure with low-rate ventilation. $\mathrm{FiO}_{2}$ was set to be 0.40 in both groups, but subsequently adjusted to arterial oxygen tension $\left(\mathrm{PaO}_{2}\right)$ and therefore different between the two groups according to 
Table 1 Characteristics, methods and results for the included studies of supplementary oxygen for trauma patients
Taher et $\mathrm{al}^{19}$
Barzilay et $\left.a\right|^{20 *}$

\begin{tabular}{ll}
\hline $\begin{array}{c}\text { Study characteristics } \\
\text { Setting }\end{array}$ & Emergency ward \\
Period & 2014 \\
\hline $\begin{array}{l}\text { Geographical location } \\
\text { Methods }\end{array}$ & Hamadan, Iran \\
\hline Aim & '...to assess the effects of normobaric hyperoxia on \\
& clinical neurological outcomes of patients with severe \\
& TBls.'
\end{tabular}

General ICU

January 1981-January 1984

Afula, Israel

'...compare the results using ventilatory method, which combines HFPPV [highfrequency positive-pressure ventilation] and low-rate conventional mechanical ventilation (LRCMV), to the results using conventional mechanical ventilation (CMV) with PEEP.'

$\begin{array}{ll}\begin{array}{l}\text { Blinding } \\ \text { Study design }\end{array} & \begin{array}{l}\text { Double blinded. } \\ \text { Inclusion criteria }\end{array} \\ & \begin{array}{l}\text { Randomised controlled trial. } \\ \text { haemodynamic stability; GCS 3-8. }\end{array} \\ \text { Exclusion criteria } & \begin{array}{l}\text { Pregnancy; chronic disease such as diabetes mellitus, } \\ \text { ischaemic heart disease, renal failure, acute pulmonary } \\ \text { oedema, history of massive myocardial infarction and } \\ \text { heart failure; blood pressure <90/60 mm Hg; successful } \\ \text { CPR; death or loss to follow-up; patients in the control } \\ \text { group in which oxygen therapy was inevitable. }\end{array}\end{array}$
Not reported.

Interventional, non-randomised. All patients admitted to the ICU with a diagnosis of severe respiratory insufficiency due to flail chest or pulmonary contusion.

Not reported.

\begin{tabular}{|c|c|c|c|c|}
\hline & Intervention group & Control group & Intervention group & Control group \\
\hline \multicolumn{5}{|l|}{ Results } \\
\hline No of patients & 34 & 34 & 11 & 10 \\
\hline Age (years), mean (SD) & $39.7(14.1)$ & 45.7 (13.3) & $40.6(22.45)$ & $39.8(18.18)$ \\
\hline Female sex, n (\%) & $9(26.5)$ & $11(32.4)$ & Not reported & Not reported \\
\hline GCS on admission, mean (SD) & $7.4(0.79)$ & $7.4(0.89)$ & & \\
\hline $\mathrm{FiO}_{2}$, mean (SD) & 0.80 & 0.50 & $0.45 \dagger$ & $0.60 \dagger$ \\
\hline $\mathrm{PaO}_{2}(\mathrm{~mm} \mathrm{Hg})$, mean (SD) & Not reported & Not reported & $89.91 \pm 10.24 \dagger$ & $78.43 \pm 11.13 \dagger$ \\
\hline \multicolumn{5}{|l|}{ Outcome measures } \\
\hline 30-Day all-cause mortality, n (\%) & $0(0)$ & $0(0)$ & $0(0)$ & $2(20)$ \\
\hline Hospital LOS (days) & $11.4(5.4)$ & $13.9(8.1)$ & Not reported & Not reported \\
\hline ICU LOS (days) & $9.4(6.6)$ & $11.4(8.4)$ & Not reported & Not reported \\
\hline $\begin{array}{l}\text { Days on mechanical ventilation, } \\
\text { mean (SD) }\end{array}$ & Not reported & Not reported & $4.2(0.91)$ & $6.1(0.8)$ \\
\hline
\end{tabular}

*This study did not strictly meet the inclusion criteria; however, it was included for descriptive purposes.

†During the first 48 hours in hospital ( $\mathrm{FiO}_{2}$ estimated from other results).

CPR, cardiopulmonary resuscitation; $\mathrm{FiO}_{2}$, inspiratory oxygen fraction; GCS, Glasgow Coma Scale Score; ICU, intensive care unit; LOS,

length of stay; $\mathrm{PaO}_{2}$, arterial oxygen tension; PEEP, positive end expiratory pressure; TBI, traumatic brain injury.

the results. Eleven patients in the intervention group received an estimated mean $\mathrm{FiO}_{2}$ of 0.45 and had a mean $\mathrm{PaO}_{2}$ of $89.91 \pm 10.24 \mathrm{~mm} \mathrm{Hg}$ during the first 48 hours after hospital admission. The control group consisted of 10 similar patients receiving an estimated mean $\mathrm{FiO}_{2}$ of 0.60 and had a mean $\mathrm{PaO}_{2}$ of $78.43 \pm 11.13 \mathrm{~mm} \mathrm{Hg}$ during the first 48 hours after hospital admission. Neither of these $\mathrm{FiO}_{2} \mathrm{~s}$ were reported in detail, but can be estimated from the data provided in the article. No simple relationship was found between the estimated $\mathrm{FiO}_{2}$ and $\mathrm{PaO}_{2}$ values presumably as a consequence of the two different ventilation strategies. Outcomes relevant to this systematic review were days on mechanical ventilation and mortality. The study found no statistically significant difference in days on mechanical ventilation between the intervention group and the control 
Table 2 Risk of bias assessment for the two included studies

\begin{tabular}{|c|c|c|c|c|}
\hline \multirow[b]{2}{*}{ Risk of bias domain } & \multicolumn{2}{|l|}{ Taher et $a^{19}$} & \multicolumn{2}{|c|}{ Barzilay et $a l^{20 *}$} \\
\hline & Judgement & Support for judgement & Judgement & Support for judgement \\
\hline $\begin{array}{l}\text { Random sequence } \\
\text { generation (selection } \\
\text { bias) }\end{array}$ & Unclear & $\begin{array}{l}\text { Quote: '....patients were divided } \\
\text { in two groups...'. } \\
\text { Comment: Not a random } \\
\text { component in the sequence } \\
\text { generation process. }\end{array}$ & Unclear & $\begin{array}{l}\text { Comment: No description of a random } \\
\text { component in the sequence generation } \\
\text { process. }\end{array}$ \\
\hline $\begin{array}{l}\text { Blinding of participants } \\
\text { and personnel } \\
\text { (performance bias) }\end{array}$ & Low & $\begin{array}{l}\text { Quote: 'In this double blind } \\
\text { clinical trial...' } \\
\text { Comment: Probably done. }\end{array}$ & Low & $\begin{array}{l}\text { Comment: No blinding is described, but } \\
\text { the relevant outcomes are not likely to } \\
\text { be influenced by lack of blinding. }\end{array}$ \\
\hline $\begin{array}{l}\text { Incomplete outcome } \\
\text { data (attrition bias) }\end{array}$ & Low & $\begin{array}{l}\text { Comment: Outcome is reported } \\
\text { for all included patients. }\end{array}$ & Unclear & $\begin{array}{l}\text { Comment: The outcomes are not } \\
\text { described as being defined before } \\
\text { commencing the study. }\end{array}$ \\
\hline $\begin{array}{l}\text { Selective reporting } \\
\text { (reporting bias) }\end{array}$ & Unclear & $\begin{array}{l}\text { Comment: No protocol is } \\
\text { available and the reported } \\
\text { outcomes are not prespecified in } \\
\text { the methods section. }\end{array}$ & Unclear & $\begin{array}{l}\text { Comment: As outcomes are not } \\
\text { described as being defined before } \\
\text { commencing the study, there is } \\
\text { insufficient information to assess this } \\
\text { domain. }\end{array}$ \\
\hline Other bias & Unclear & $\begin{array}{l}\text { Comment: There is insufficient } \\
\text { information on the study design } \\
\text { to assess whether an important } \\
\text { risk of bias exists. }\end{array}$ & High & $\begin{array}{l}\text { Quote: 'Those in the study group were } \\
\text { connected to a two-ventilator HFPPV } \\
\text { system of our own design'. } \\
\text { Comment: The authors are likely to have } \\
\text { a preference for their own design. }\end{array}$ \\
\hline
\end{tabular}

*This study did not strictly meet the inclusion criteria, however, it was included for descriptive purposes.

HFPPV, high-frequency positive-pressure ventilation.

group (4.2 days (SD: 0.91) vs 6.1 days (SD: 0.8), respectively, $\mathrm{p}<0.1)$. In terms of mortality, two $(20 \%)$ patients in the control group died compared with none in the intervention group. The $\mathrm{p}$ value was not reported, but the difference was not statistically significant using Fisher's exact test.

The risk of bias assessment for the included studies is presented in table 2 . In the study by Taher $e t$ al, three domains were judged to have a low risk of bias (blinding of participants and personnel, blinding of outcome assessment, incomplete outcome data), none to have a high risk of bias, and four domains to have an unclear risk of bias (random sequence generation, allocation concealment, selective reporting, other bias). The study by Barzilay et al was judged to have two domains with low risk of bias (blinding of participants and personnel, blinding of outcome assessment), two domains with high risk of bias (allocation concealment, other bias) and three domains with an unclear risk of bias (random sequence generation, incomplete outcome data, selective reporting).

\section{DISCUSSION}

\section{Summary of evidence}

In this systematic review of interventional trials of the use of supplementary oxygen in the initial treatment of trauma patients, we identified no studies of spontaneously breathing patients, and only one interventional trial of intubated trauma patients was found to fulfil the inclusion criteria. Taher et $a l^{19}$ found the low $\mathrm{FiO}_{2}$ group $(0.50)$ to have slightly longer LOS in hospital and LOS in ICU than the high- $\mathrm{FiO}_{2}$ group (0.80); however, these differences were not statistically significant. Additionally, no patient died in either group. In another study by Barzilay et $a l,{ }^{20}$ which did not strictly fulfil the inclusion criteria, no statistically significant differences were found between the groups, although patients in the high- $\mathrm{FiO}_{2}$ group (0.60) tended to have a higher mortality and more days on mechanical ventilation than the patients in the low- $\mathrm{FiO}_{2}$ group (0.45). Due to the low number and heterogeneity of the included studies, we neither found it possible to pool the results of the two studies, nor to draw any conclusions from these findings. 
The rationale for supplementation of oxygen for various patient groups has for decades-and even centuries-seemed self-evident for most healthcare providers. ${ }^{21}$ Oxygen supplementation, often in excess, has been considered a safe measure rather than an intervention that could potentially be harmful and thus needing a clear indication of administration. Supplementation of oxygen has, until recently, escaped the critical evaluation of its value and indication as is necessary for all other drugs not having the same historical, 'self-evident' benefit as is the case for oxygen. As previously described, trauma patient management is mostly based on guideline recommendations including rather liberal and non-specific oxygen supplementation. Thus, it seems surprising that, even though supplementary oxygen is widely used in the treatment of trauma patients and included in international trauma guidelines, this systematic review finds that the evidence for the use of supplementary oxygen for spontaneously breathing trauma patients is non-existing, and for mechanically ventilated trauma patients the evidence is extremely limited and of low quality. In an era of evidence-based medicine these findings seem inappropriate, and we cannot continue to avoid investigating the potential benefits and harms of a drug that is so widely used.

Supplementary oxygen increases the $\mathrm{PaO}_{2}$ of oxygen in the alveoli, thus increasing the oxygen gradient across the alveolar-capillary membrane. This is likely to increase the $\mathrm{PaO}_{2}$ when oxygenation is impeded by a barrier in the transport of oxygen across the alveolar-capillary membrane. However, that is not common in trauma patients. On the other hand, it can be reasonable to administer supplementary oxygen in order to increase the amount of oxygen in the lungs to prolong the safe apnoea time. ${ }^{22}$

Both hypoxaemia and hyperoxaemia may be harmful. Hypoxaemia may cause hypoxic neuronal cell death leading to irreversible brain damage, whereas hyperoxaemia has been found to increase the risk of pulmonary complications like the formation of atelectases and airway inflammation. ${ }^{23}$

The effect of hyperoxia on outcomes following TBI has been investigated in a few retrospective studies. Rincon et $a l^{24}$ and Brenner et $a l^{25}$ assessed short-term outcomes and they both found hyperoxia to be associated with increased in-hospital mortality compared with normoxia. Additionally, Brenner et al found that hyperoxia was associated with lower GCS scores at discharge. Another retrospective study by Davis $e t a t^{26}$ of patients with moderate to severe TBI found both hypoxaemia and hyperoxaemia to be correlated with decreased survival to discharge compared with patients with normoxia. In contrast, Raj et $a l^{27}$ detected no association between hyperoxaemia and 6-month mortality.

The evidence for the use of supplementary oxygen has been investigated in recently published systematic reviews. In a Cochrane review from 2015, Wetterslev et $a l^{10}$ included 28 studies and found no association between perioperative $\mathrm{FiO}_{2}$ (high: $0.60-0.90$ vs low: $0.30-0.40$ ) and postoperative surgical site infection and mortality. In another Cochrane review of supplementary oxygen for patients with suspected or confirmed acute myocardial infarction (AMI), Cabello $e t a l^{28}$ included five studies, and they were not able to draw conclusions for or against the use of supplementary oxygen for patients with AMI. Hyperoxia in postreturn of spontaneous circulation CA patients has been studied in a systematic review and meta-analysis by Wang et al. ${ }^{9}$ Fourteen studies were included, and the authors found hyperoxia to be correlated with increased in-hospital mortality in a meta-analysis of eight of the included studies. Finally, Damiani et $a l^{7}$ have looked at the association between arterial hyperoxia and mortality for adult ICU patients (mechanically ventilated, post-CA, stroke, TBI) in a systematic review and meta-analysis from 2014 of 17 studies. In the meta-analysis, hyperoxia was associated with increased mortality for patients post-CA, stroke and TBI, though the authors report the studies to be rather heterogeneous. As the trauma population is a very heterogeneous and typically a younger and less comorbid group of patients than other critically ill populations (ie, AMI, CA, stroke), the results of the before-mentioned systematic reviews of other patient populations cannot be extrapolated to the trauma population. However, there seems to be an implication that treatment with excess oxygen and hyperoxia can be harmful or at least not beneficial. This, again, stresses the need for investigating the effects of supplementary oxygen and cases of hyperoxia in the trauma population.

\section{Strengths and limitations}

This systematic review was conducted in accordance with the PRISMA guidelines ${ }^{14}$ ensuring a systematic and internationally accepted methodological approach. The strengths of this approach include predefined PICOS criteria used to assess for study eligibility, the use of a wide search string in multiple databases, a structured screening and inclusion process by two independent authors, and data collection and risk of bias assessment by the same two independent authors using predetermined forms. Our study is limited by the weaknesses of a systematic review in general: the possibility of missing unpublished studies which creates a potential publication bias, and the possibility that we did not identify all relevant studies despite our systematic methodology. The patient population we included was defined in rather general terms (ie, adult trauma patients) which may have increased the heterogeneity of the studies; however, we found this to be necessary in order to increase the clinical relevance of our findings. We wanted to study the initial treatment phase of trauma patients and chose this to be the first 24 hours after the traumatic incident. This time cut-off was chosen rather arbitrarily and did exclude one potentially eligible study. ${ }^{29}$ As per our inclusion criteria for this systematic review, we wanted to include both prehospital and in-hospital studies; however, both included studies investigated in-hospital patients with no data on the prehospital supplementary oxygen treatment. As a large proportion of trauma patients receive prehospital supplementary oxygen, ${ }^{56}$ it is a limitation not to know whether the per protocol $\mathrm{FiO}_{2}$-group allocation is the only oxygenation 
treatment the patient has received since the traumatic incident.

The study by Barzilay et al was included in the review despite lacking strict adherence to the inclusion criteria. We chose to do this, as evidence in this field proved to be extremely sparse, and we wished to report as much of the existing evidence as possible.

We were only able to include two small studies of mechanically ventilated trauma patients, and two different methods of mechanical ventilation were used in the study by Barzilay et al. Thus, the studies were not suitable for pooling results, and we were neither able to draw any conclusions nor provide recommendations for the $\mathrm{FiO}_{2}$ for mechanically ventilated trauma patients. Furthermore, as no studies of spontaneously breathing trauma patients were found, we cannot provide recommendations for the use of supplementary oxygen for spontaneously breathing trauma patients either.

\section{CONCLUSIONS}

In this systematic review of supplementary oxygen for trauma patients in the initial phase of treatment, we identified no interventional trials including spontaneously breathing trauma patients and only two small low-quality studies assessing oxygen fraction in intubated trauma patients. Thus, the current practice of liberal oxygen administration must be questioned, and interventional studies of supplementary oxygen should be conducted in trauma patients.

Contributors TGE, JSB, JS and LSR have contributed to conception and design of the study. TGE and JSB have contributed to the acquisition of data. TGE, JSB, JS and LSR have contributed to the analysis and interpretation of data. TGE, JSB, JS and LSR have participated in drafting and revising the manuscript critically. TGE, JSB, JS and LSR have given their final approval of the manuscript to be submitted.

Funding Our research group is supported by the Tryg Foundation, however, this research received no specific grant from any funding agency in the public, commercial or not-for-profit sectors.

Competing interests None declared.

Patient consent Not required.

Provenance and peer review Not commissioned; externally peer reviewed. Data sharing statement Not applicable for a systematic review.

Open access This is an open access article distributed in accordance with the Creative Commons Attribution Non Commercial (CC BY-NC 4.0) license, which permits others to distribute, remix, adapt, build upon this work non-commercially, and license their derivative works on different terms, provided the original work is properly cited and the use is non-commercial. See: http://creativecommons.org/ licenses/by-nc/4.0/

(c) Article author(s) (or their employer(s) unless otherwise stated in the text of the article) 2018. All rights reserved. No commercial use is permitted unless otherwise expressly granted.

\section{REFERENCES}

1. ACo S. ATLS Student Course Manual: Advanced Trauma Life Support. 9 edn, 2012.

2. Mortality GBD CoDC, CoDC M. GBD 2013 Mortality and Causes of Death Collaborators. Global, regional, and national age-sex specific all-cause and cause-specific mortality for 240 causes of death, 19902013: a systematic analysis for the Global Burden of Disease Study 2013. Lancet 2015;385:117-71.
3. PHTLS. Basic and Advanced Prehospital Trauma Life Support. 5 edn. Mosby, 2003.

4. Hale KE, Gavin C, O'Driscoll BR. Audit of oxygen use in emergency ambulances and in a hospital emergency department. Emerg Med J 2008;25:773-6.

5. McMullan J, Rodriquez D, Hart KW, et al. Prevalence of prehospital hypoxemia and oxygen use in trauma patients. Mil Med 2013;178:1121-5.

6. Stockinger ZT, Mcswain NE. Prehospital supplemental oxygen in trauma patients: its efficacy and implications for military medical care. Mil Med 2004;169:609-12.

7. Damiani E, Adrario E, Girardis M, et al. Arterial hyperoxia and mortality in critically ill patients: a systematic review and metaanalysis. Crit Care 2014;18:711.

8. Helmerhorst HJ, Roos-Blom MJ, van Westerloo DJ, et al. Association Between Arterial Hyperoxia and Outcome in Subsets of Critical IIIness: A Systematic Review, Meta-Analysis, and Meta-Regression of Cohort Studies. Crit Care Med 2015;43:1508-19.

9. Wang $\mathrm{CH}$, Chang WT, Huang $\mathrm{CH}$, et al. The effect of hyperoxia on survival following adult cardiac arrest: a systematic review and metaanalysis of observational studies. Resuscitation 2014;85:1142-8.

10. Wetterslev J, Meyhoff CS, Jørgensen LN, et al. The effects of high perioperative inspiratory oxygen fraction for adult surgical patients. Cochrane Database Syst Rev 2015;25:CD008884.

11. Belda FJ, Aguilera L, García de la Asunción J, et al. Supplemental perioperative oxygen and the risk of surgical wound infection: a randomized controlled trial. JAMA 2005;294:2035-42.

12. Greif R, Akça O, Horn EP, et al. Supplemental perioperative oxygen to reduce the incidence of surgical-wound infection. N Engl J Med 2000;342:161-7.

13. GSe HJPT. Cochrane Handbook for Systematic Reviews of Interventions Version 5.1.0: The Cochrane Collaboration, 2011.

14. Moher D, Liberati A, Tetzlaff J, et al. Preferred reporting items for systematic reviews and meta-analyses: the PRISMA statement. BMJ 2009;339:b2535.

15. Moher D, Shamseer L, Clarke $M$, et al. Preferred reporting items for systematic review and meta-analysis protocols (PRISMA-P) 2015 statement. Syst Rev 2015;4:1.

16. PROSPERO. International prospective register of systematic reviews. https://www.crd.york.ac.uk/PROSPERO/.

17. Covidence systematic review software. Veritas Health Innovation. Melbourne. www.covidence.org.

18. Higgins JPT AD, Sterne JAC. Assessing risk of bias in included studies. In: Higgins JPT, Green S, Cochrane Handbook for Systematic Reviews of Interventions Version 5.1.0: The Cochrane Collaboration, 2011.

19. Taher A, Pilehvari Z, Poorolajal J, et al. Effects of Normobaric Hyperoxia in Traumatic Brain Injury: A Randomized Controlled Clinical Trial. Trauma Mon 2016;21:e26772.

20. Barzilay E, Lev A, Ibrahim M, et al. Traumatic respiratory insufficiency: comparison of conventional mechanical ventilation to high-frequency positive pressure with low-rate ventilation. Crit Care Med 1987;15:118-21.

21. Kelly C. Oxygen therapy: time to move on? Ther Adv Respir Dis 2014;8:191-9.

22. Edmark L, Kostova-Aherdan K, Enlund M, et al. Optimal oxygen concentration during induction of general anesthesia. Anesthesiology 2003;98:28-33

23. Helmerhorst HJ, Schultz MJ, van der Voort PH, et al. Bench-tobedside review: the effects of hyperoxia during critical illness. Crit Care 2015;19:284.

24. Rincon F, Kang J, Vibbert M, et al. Significance of arterial hyperoxia and relationship with case fatality in traumatic brain injury: a multicentre cohort study. J Neurol Neurosurg Psychiatry 2014:85:799-805.

25. Brenner M, Stein D, Hu P, et al. Association between early hyperoxia and worse outcomes after traumatic brain injury. Arch Surg 2012;147:1042-6.

26. Davis DP, Meade W, Sise MJ, et al. Both hypoxemia and extreme hyperoxemia may be detrimental in patients with severe traumatic brain injury. J Neurotrauma 2009;26:2217-23.

27. Raj R, Bendel S, Reinikainen M, et al. Hyperoxemia and long-term outcome after traumatic brain injury. Crit Care 2013;17:R177.

28. Cabello JB, Burls A, Emparanza JI, et al. Oxygen therapy for acute myocardial infarction. Cochrane Database Syst Rev 2016;12:Cd007160.

29. Stall A, Paryavi E, Gupta R, et al. Perioperative supplemental oxygen to reduce surgical site infection after open fixation of high-risk fractures: a randomized controlled pilot trial. $J$ Trauma Acute Care Surg 2013;75:657-63. 